\title{
Near Earth Objects Research in Pulkovo Observatory
}

\author{
A. V. Devyatkin, E. A. Bashakova, D. L. Gorshanov, A. V. Ivanov, \\ S. V. Karashevich, V. V. Kouprianov, V. N. L'vov, K. N. Naumov, \\ E. S. Romas, V. Yu. Slesarenko, N. A. Shakht, E. N. Sokov, \\ S. D. Tsekmeister, O. O. Vasilkova and I. A. Vereschagina
}

Pulkovo Astronomical Observatory of Russian Academy of Science email: olyaov@mail.ru

More than 20000 observations of Near Earth asteroids and comets are collected and reduced in Pulkovo Observatory during last 10 years. For observations of these objects two robotic telescopes are used - ZA-320M (Cassegrain system, $D=320 \mathrm{~mm}, F=3200$ $\mathrm{mm}$ ) at Pulkovo and MTM-500M (Maksutov - Cassegrain system, $D=500 \mathrm{~mm}, F=$ $4100 \mathrm{~mm}$ ) at Kislovodsk mountain station. These telescopes perform CCD observations of objects up to 18.0 and 20.5 magnitude, correspondingly. The results of observations are regularly submitted to Minor Planet Center.

Two software packages developed in Pulkovo observatory are now in practice. The first one, APEX, is used for automatic CCD frame processing (Devyatkin et al. (2010)). The second, EPOS, provides independent ephemeris support, checkout of observations and estimation of their accuracy. It helps in objects identification, orbits determination and improvement, modeling the objects apparent motion on the sky and orbital motion in space (L'vov, Tsekmeister (2012)). Its recent facility - studying the motion of Troyan, Horseshoe and Quasi-satellite asteroids.

Several NEAs were observed in the frame of GAIA FUN-SSO program.

The Near Earth asteroid 2005 YU55 has approached to Venus, Earth and Mars. The EPOS software helped to model the sharp changes of its orbit during the close approaches. It was observed with ZA-320M and MTM-500M telescopes during its close approach to the Earth in November 2012. 926 positions with mean accuracies of $0 " .1-0 " .4$ were obtained, results were sent to Minor Planet Center. Using the observations the asteroid orbit was improved. From its light-curves the period of rotation was estimated and the color-indices in BVRI system were determined. To estimate the period (using Scargles method) all our observations which were made without filter were processed. The value of $16.3 \mathrm{hr}$ was obtained, while the value determined earlier with radar observations is 18 hr (see http://ssd.jpl.nasa.gov/sbdb.cgi?sstr=308635).

During processing the data, one more period was noticed in observations made with both telescopes in several nights. Its value varies from 0.9 to $1.2 \mathrm{hr}$ for different nights. The magnitude range is about $0.15^{\mathrm{m}}$. The long-time observations $(\approx 10 \mathrm{hr})$ include several period durations. If this period is real, it could meant that the asteroid is binary.

The weighted means of color-indices from our observations are the following: $B-V=$ $0.67^{m} \pm 0.07^{m}, V-R=0.34^{m} \pm 0.09^{m}, R-I=0.30^{m} \pm 0.07^{m}$. Our value of $V-R$ color-index is in agreement with the effective color $\left(V-R=0.37^{m}\right)$ measured from spectrum of the asteroid in works Hicks et al. (2011a), Hicks et al. (2011b).

We have tried to classify 2005 YU55 asteroid using our wide-band photometry. Dandy et al. (2003) classify asteroids on Tholens classification using wide-band photometry: $B V R I$ and additional $Z$ band at $0.91 \mu \mathrm{m}$. We have no $Z$ filter. But using their method 
in this narrower $(0.44-0.83 \mu \mathrm{m})$ spectral diapason, the spectrum form of the asteroid is close to B, F, C, G (Tholen) classes - the classes with flat spectra in visible range. B class is the closest.

Hicks et al. (2011b) have built phase curve for 2005 YU55 asteroid in $R$ band and determined its absolute magnitude and slope parameter. We put our $R$ band observations into the sketch from this work. The observations lay on the same phase curve but have greater scattering. They are made with lower phase angles than in work Hicks et al. (2011b) and confirm the values of absolute magnitude and slope parameter of the asteroid.

Another asteroid observed in the frame of GAIA FUN-SSO program is TP3522. On January 20, 2013, we made 24 observations of this faint (about $20^{m}$ ) asteroid with MTM$500 \mathrm{M}$ telescope. These observations amount about $1 / 3$ of all observations for the object in MPC database (77 observations). Mean accuracy of our observations is 0".2 in each coordinate. These observations (along with observations of other observatories) confirmed discovery of the asteroid and allowed to improve its orbit.

The asteroid 2008 TC3 was observerd at Pulkovo with ZA-320M telescope. The collision of 2008 TC3 with Earth (in 19 hours after its discovery) was predicted, presumably, in Northern Sudan. About 800 observations were obtained in Pulkovo which amount about $1 / 3$ of all observations available in MPC. Based on the analysis of these observations, the following physical parameters of asteroid were estimated: its absolute magnitude, $M=30.6 \pm 0.4^{m}$; size, $4.8 \pm 0.8 \mathrm{~m}$; weight, $131 \pm 5 \mathrm{t}$; and rotation period of $48.6 \pm 0.6$ sec. The trajectory of the asteroid was computed, as well as the place of its collision with the Earth (for the case when explosion does not occur). The point of explosion and place of debris fall correlate with computed trajectory.

For potentially hazardous asteroid 2009 WZ104, more than 1000 observations were made at Pulkovo. Using these, orbit of the asteroid was improved and color-indices were obtained: $B-V=0.65^{m} \pm 0.7^{m}, V-R=0.18^{m} \pm 0.05^{m}, R-I=-0.26^{m} \pm 0.07^{m}$. From its light curve, 2 periods were determined: $P_{1}=0.4$ and $P_{2}=2.76$ days.

With MTM-500M telescope, several faint $\left(>20^{m}\right)$ asteroids were discovered $(2010$ UP67, 2010 XL46, 2010 XM46, 2010 XA15) and rediscovered (2010 SY11 = 2004 TR356, 2010 VX29 $=2008$ EG30, C20N117 = 2008 FM60, NA12044=2005 XP17).

\section{References}

Dandy, C. L. et al. 2003, Icarus 163, 363

Devyatkin, A. V. et al. 2010, Solar System Research 44, 66

Hicks, M. et al. 2011a, The Astronomer's Telegram 2571

Hicks, M. et al. 2011b, The Astronomer's Telegram 3763

L'vov, V. N. \& Tsekmeister, S. D. 2012, Solar System Research 46, 177 